in vivo $34: 2571-2576(2020)$

doi:10.21873/invivo.12074

\title{
Older Age Is Associated With Better Compliance With Follow-up in Taiwan After Functional Endoscopic Sinus Surgery
}

\author{
TAMMY TSAI ${ }^{1 *}$, LIANG-CHUN SHIH ${ }^{1,2^{*}}$, IVAN T. LEE ${ }^{3}$, TEIK-YING NG ${ }^{1,4}$, JONG-YI WANG $^{5}$, \\ CHE-LUN HSU ${ }^{1}$, DA-TIAN BAU ${ }^{2,6,7}$ and CHIH-JAAN TAI ${ }^{1,4,5}$ \\ ${ }^{1}$ Department of Otorhinolaryngology, China Medical University Hospital, Taichung, Taiwan, R.O.C.; \\ ${ }^{2}$ Graduate Institute of Biomedical Sciences, China Medical University, Taichung, Taiwan, R.O.C.; \\ ${ }^{3}$ Division of Allergy, Immunology, and Rheumatology, Department of Pediatrics, \\ Stanford University School of Medicine, Stanford, CA, U.S.A.; \\ ${ }^{4}$ School of Medicine, China Medical University, Taichung, Taiwan, R.O.C.; \\ ${ }^{5}$ Department of Health Services Administration, China Medical University, Taichung, Taiwan, R.O.C.; \\ ${ }^{6}$ Terry Fox Cancer Research Laboratory, Department of Medical Research, \\ China Medical University Hospital, Taichung, Taiwan, R.O.C.; \\ ${ }^{7}$ Department of Bioinformatics and Medical Engineering, Asia University, Taichung, Taiwan, R.O.C.
}

\begin{abstract}
Background/Aim: Functional endoscopic sinus surgery (FESS) is frequently conducted for restoring sinus ventilation and function. Postoperative care is critical for success. However, loss to follow-up is disturbing. The specific aim of this study was to identify critical factors contributing to loss of patients to follow-up and how to improve it. Patients and Methods: A total of 221 patients with chronic rhinosinusitis undergoing FESS were enrolled. Patients were divided into three groups according to their follow-up after surgery: Less than 1 month (short-term), 13 months (medium-term) and more than 3 months (longterm). The gender, age, smoking status, comorbidities, laterality, Lund-Mackay score, 22-question Sinonasal Outcome Test, nasal obstruction Visual Analogue Scale and mucociliary clearance were evaluated for their contribution
\end{abstract}

This article is freely accessible online.

*These Authors contributed equally to this study.

Correspondence to: Da-Tian Bau, Terry Fox Cancer Research Laboratory, Department of Medical Research, China Medical University Hospital, Tel: +886 422053366 Ext. 5805, e-mail: datian@mail.cmuh.org.tw/artbau2@gmail.com; Chih-Jaan Tai, Department of Otorhinolaryngology, China Medical University Hospital, 2 Yuh-Der Road, Taichung, 404 Taiwan, R.O.C. E-mail: edsam@seed.net.tw

Key Words: Adult rhinology, age, functional endoscopic sinus surgery, Taiwan. to better compliance in follow-up. Results: The results revealed that older patients had better compliance in followup compared with younger ones $(p=0.0093)$. Other factors were not contributory $(p>0.05)$. Conclusion: In contrast to the US, older patients in Taiwan have better compliance in postoperative follow-up, while younger ones require more education on the importance of follow-up.

Functional endoscopic sinus surgery (FESS) is a minimally invasive procedure that aims to recover sinus ventilation and sinus mucociliary function. It is indicated for patients who have complicated acute rhinosinusitis or chronic rhinosinusitis (CRS) (1-3). Successful FESS creates an accessible and open sinus cavity that allows for continuous topical medical therapy (4). Complementary to FESS, postoperative care is important in the management of CRS and is highly recommended by international leaders $(4,5)$. Although there is no standardized regimen and the optimal timing remains controversial $(6,7)$, postoperative care is important for long-term success of treating CRS, including minimizing avoidable complications and optimizing longterm outcomes $(4,8,9)$. The purposes of postoperative care include promoting surgical wound healing, optimizing mucosa regeneration and restoration of mucociliary function, reducing local infection and inflammation, improving shortterm patient symptoms, and minimizing complications in the early postoperative phase (4). However, poor compliance with postoperative follow-up visits remains a common issue. Patients who do not have regular follow-up are at risk of recurrent rhinosinusitis and may need revision FESS (10). In 
in vivo $34: 2571-2576(2020)$

Table I. Univariate comparison of patient follow-ups according to follow-up appointments according to gender, age and smoking habit.

\begin{tabular}{llcccc}
\hline & & Overall & $\begin{array}{c}<1 \text { Month } \\
\text { (Short-term) }\end{array}$ & $\begin{array}{c}1-3 \text { Months } \\
\text { (Medium-term) }\end{array}$ & $\begin{array}{c}>3 \text { Months } \\
\text { (Long-term) }\end{array}$ \\
\hline Patients, $n$ & Total & 221 & $39(17.6 \%)$ & $94(42.5 \%)$ & $88(39.8 \%)$ \\
Gender (\%) & Male & $129(58.4 \%)$ & $25(64.1 \%)$ & $57(60.6 \%)$ & $47(53.4 \%)$ \\
Age, years & Mean \pm SD & $45.10 \pm 15.05$ & $43.31 \pm 15.45$ & $42.44 \pm 14.60$ & $48.73 \pm 14.78$ \\
Smokers, n $(\%)$ & Total & $55(24.9 \%)$ & $14(35.9 \%)$ & $25(26.6 \%)$ & $16(18.2 \%)$ \\
\hline
\end{tabular}

Data are means $\pm \mathrm{SD}$. Statistically significant $p$-values are shown in bold.

addition, reduced follow-up may limit the assessment of long-term clinical outcome and hamper future research into improved therapy for CRS (11).

Non-compliance with scheduled follow-up clinic visits have been well studied in other fields of medicine such as in general surgery, orthopedic surgery, and emergency medicine (11). Although postoperative care is often considered as important as FESS itself, as of now, to our knowledge there are few published studies regarding patient characteristics that lead to loss to follow-up after FESS in patients with CRS. Therefore, the aim of this study was to identify the risk factors associated with loss to follow-up after FESS in patients with CRS.

\section{Patients and Methods}

Patients. This case-control study recruited patients with chronic rhinosinusitis who underwent primary FESS between January 2017 and May 2018 at China Medical University Hospital in Taichung, Taiwan. All patients had undergone FESS at least 3 months prior to the start of this study. Patients who had revision FESS before January 2017 were excluded. All patients received verbal and written instructions for clinic follow-up at 1 week, 2 weeks, 1 month, and 3 months after FESS, and were categorized into three groups according to their compliance with postoperative follow-up: Short-term follow-up, consisting of patients who presented to one or no follow-up appointments; medium-term follow-up, consisting of patients who presented to two follow-ups; long-term follow-up, consisting of patients who presented to all three follow-ups.

The variables included in this study were sex, age, smoking status, laterality of sinus disease, Lund-Mackay scoring (12), atopic comorbidities, pre-operative 22-question Sinonasal Outcome Test (SNOT-22) (13), and mucociliary clearance (MCC) (14). The SNOT-22 score was analyzed in total as well as by its five specific symptom domains (15). We also included nasal obstruction Visual Analogue Scale (VAS), which ranges from 1 to 5 for each side [1 for no nasal obstruction $(<20 \%), 2$ for mild nasal obstruction (20$40 \%), 3$ for moderate nasal obstruction (40-60\%), 4 for severe nasal obstruction (60-80\%), and 5 for total nasal obstruction $(>80 \%)]$. The scores of bilateral sides were tabulated together. All variables above were analyzed amongst the three groups.

Statistical analysis. Patient demographics (including sex, age, smoking status), sinusitis characteristics (laterality), co-morbidity of atopy (allergic rhinitis, asthma, atopic dermatitis), preoperative
Lund-Mackay score, preoperative SNOT-22, MCC, and nasal obstruction VAS, were compared amongst the three groups using typical Pearson's chi-squared test. All tests were set at a significance level of $p<0.05$. Statistics were performed using SPSS Statistics ${ }^{\circledR}$ version 24 (IBM, Armonk, NY, USA).

\section{Results}

A total of 221 patients met the eligibility criteria for this study and their characteristics are summarized in Table I. The average age of eligible patients was 45.1 years. There were 129 male and 92 female patients (Table I). Regarding the follow-up pattern, $214(96.8 \%)$ patients presented to the postoperative clinic appointment at 2 weeks after the operation, $182(82.4 \%)$ patients presented at the 1-month follow-up post-FESS, and only 88 (39.8\%) patients presented for the follow-up at 3 months after FESS. The patients were divided into three groups based on their compliance with follow-up. There were 39 (17.6\%), 94 $(42.5 \%)$, and $88(39.8 \%)$ patients in the groups with short-, medium- and long-term follow-up, respectively (Table I).

Regarding the main outcome measurement of follow-up at 3 months post-surgery, a univariate analysis was performed. In the univariate analysis, age was found to be a statistically practical predictor for loss to follow-up $(p=0.0093$ ) (Table I), with older patients having better compliance in follow-up. The remaining variables, including gender and smoking status were not statistically significant (both $p>0.05$ ) (Table I).

The comorbidities after FESS may include deviated nasal septum, allergic rhinitis, asthma, eczema and atopy, which may be co-present. We are interested in the contributions of laterality, Lund-Mackay scores and these comorbidities to the compliance of follow-up. The overall comparisons of these factors among the three groups are shown in Table II. Neither comorbidities, laterality nor the Lund-Mackay scores were associated with a higher willingness in compliance after FESS (all $p>0.05$ ) (Table II).

All the patients were evaluated with SNOT-22 test before undergoing and after FESS. In Table III, the total scores, in addition to those for the five specific symptom domains are compared among the three groups. The results showed that neither the total scores $(p=0.6612)$ nor the rhinological 
Tsai et al: Better Compliance in Follow-up After Sinus Surgery

Table II. Univariate comparison of patient follow-up regarding their comorbidity, laterality, Lund-Mackay scoring and atopy.

\begin{tabular}{|c|c|c|c|c|c|}
\hline & $\begin{array}{l}\text { Overall } \\
(\mathrm{n}=221)\end{array}$ & $\begin{array}{c}<1 \text { Month } \\
(\text { Short-term) } \\
(\mathrm{n}=39)\end{array}$ & $\begin{array}{c}1-3 \text { Months } \\
\text { (Medium-term) } \\
\quad(\mathrm{n}=94)\end{array}$ & $\begin{array}{c}>3 \text { Months } \\
(\text { Long-term) } \\
(\mathrm{n}=88)\end{array}$ & $p$-Value \\
\hline DNS (\%) & $46(20.8 \%)$ & $7(17.9 \%)$ & $22(23.4 \%)$ & $17(19.3 \%)$ & 0.7060 \\
\hline $\mathrm{AR}(\%)$ & $72(32.6 \%)$ & $12(30.8 \%)$ & $33(35.1 \%)$ & $27(30.7 \%)$ & 0.7883 \\
\hline Asthma (\%) & $12(5.4 \%)$ & $1(2.6 \%)$ & $5(5.3 \%)$ & $6(6.8 \%)$ & 0.6199 \\
\hline Eczema $(\%)$ & $14(6.3 \%)$ & $3(7.7 \%)$ & $8(8.5 \%)$ & $3(3.4 \%)$ & 0.3429 \\
\hline Atopy (\%)a & $79(35.7 \%)$ & $14(35.9 \%)$ & $37(39.3 \%)$ & $28(31.8 \%)$ & 0.5694 \\
\hline Laterality $(\%) \mathrm{b}$ & $105(48.2 \%)$ & $21(53.8 \%)$ & $46(48.9 \%)$ & $38(44.7 \%)$ & 0.6269 \\
\hline Lund-Mackay score & $9.05 \pm 5.57$ & $9.59 \pm 6.23$ & $8.94 \pm 5.58$ & $8.93 \pm 5.30$ & 0.8021 \\
\hline
\end{tabular}

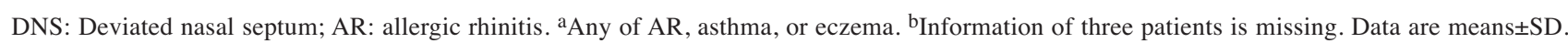

Table III. Univariate comparison of patient follow-up regarding their preoperative 22-question Sinonasal Outcome Test (SNOT-22) with five domains.

\begin{tabular}{|c|c|c|c|c|c|}
\hline & $\begin{array}{l}\text { Overall } \\
(\mathrm{n}=221)\end{array}$ & $\begin{array}{c}<1 \text { Month } \\
\text { (Short-term) } \\
(n=39)\end{array}$ & $\begin{array}{c}1-3 \text { Months } \\
\text { (Medium-term) } \\
(\mathrm{n}=94)\end{array}$ & $\begin{array}{c}>3 \text { Months } \\
(\text { Long-term) } \\
(\mathrm{n}=88)\end{array}$ & $p$-Value \\
\hline Pre-operative SNOT total & $40.95 \pm 21.68$ & $42.50 \pm 22.67$ & $41.74 \pm 21.16$ & $39.36 \pm 21.80$ & 0.6612 \\
\hline Rhinological symptoms & $13.97 \pm 6.79$ & $13.89 \pm 7.30$ & $14.68 \pm 6.97$ & $13.22 \pm 7.51$ & 0.3789 \\
\hline Extra-nasal rhinological symptoms & $7.18 \pm 4.00$ & $7.08 \pm 4.24$ & $7.52 \pm 3.83$ & $6.84 \pm 4.04$ & 0.5103 \\
\hline Ear/facial symptoms & $5.90 \pm 4.74$ & $6.00 \pm 4.05$ & $6.25 \pm 5.07$ & $5.47 \pm 4.66$ & 0.5523 \\
\hline Psychological dysfunction & $12.50 \pm 8.69$ & $13.71 \pm 8.52$ & $12.36 \pm 8.77$ & $12.11 \pm 8.70$ & 0.6237 \\
\hline Sleep dysfunction & $10.13 \pm 6.97$ & $10.68 \pm 7.21$ & $10.01 \pm 6.77$ & $10.01 \pm 7.13$ & 0.8633 \\
\hline
\end{tabular}

Data are means \pm SD.

symptoms $(p=0.3789)$, extra-nasal rhinological symptoms $(p=0.5103)$, ear/facial symptoms $(p=0.5523)$, psychological dysfunction $(p=0.6237)$, nor sleep dysfunction $(p=0.8633)$ were significantly different among the short-, medium-and long-term follow-up groups (Table III). The postoperative scores for SNOT-22 are shown in Table IV. The scores for each item are all of similar level among the three groups of patients (all $p>0.05$ ) (Table IV). Noticeably, all the scores for each item were all significantly lower than the corresponding scores in the preoperative evaluation (Tables III and IV).

The pre-operative nasal obstruction VAS on left, right, bilateral and MCC among the three groups are listed and analyzed in Table V. The results showed the differences for left, right, bilateral nasal obstruction VAS, and MCC among the three groups not to be statistically significant (all $p>0.05$ ) (Table V).

\section{Discussion}

Clinically, FESS has been recognized the gold-standard treatment for patients with complicated acute rhinosinusitis or chronic rhinosinusitis $(1,2,3,16)$. The aim of FESS is to improve ostiomeatal ventilation and drainage, to remove potential polyps and accumulation of mucopus with the preservation of normal mucosa, and to restore sinus mucociliary function $(1,3,9)$. After FESS, the timing of wound healing varies greatly from case to case. A macroscopically normal appearing mucosa usually takes 1218 weeks (that is to say about 3-4.5 months) after surgery to heal, although subepithelial changes may continue to occur even 6 months after the operation (17). Thus, one of the most important factors in determining good functional surgical outcome after FESS is meticulous postoperative follow-up care (4). An unsuccessful FESS outcome may lead to significant economic, emotional and physical issues for the patient $(2,18)$. Despite its importance, as far as we are aware, very few studies have been published focused on risk factors associated with loss to follow-up after FESS.

After FESS, patients frequently miss one or two followup appointments after discharge from hospitals. Besides poor functional outcomes, reduced rates of follow-up may limit assessments of the patient's long-term clinical condition. If patients were free from symptoms after the operation, the success of FESS may be underestimated. On the other hand, if the patients had an unsuccessful surgical outcome but did not present for follow-up, the success of FESS may be overestimated (11). In any case, patients should be followedup regularly at least 3 months after FESS. 
in vivo $34: 2571-2576(2020)$

Table IV. Univariate comparison of patient follow-up regarding each item of the 22-question Sinonasal Outcome Test (SNOT-22).

\begin{tabular}{|c|c|c|c|c|c|}
\hline $\begin{array}{l}\text { SNOT-22 } \\
\text { question }\end{array}$ & $\begin{array}{l}\text { Overall } \\
(n=221)\end{array}$ & $\begin{array}{c}<1 \text { Month } \\
\text { (Short-term) } \\
(\mathrm{n}=39)\end{array}$ & $\begin{array}{c}\text { 1-3 Months } \\
\text { (Medium-term) } \\
(\mathrm{n}=94)\end{array}$ & $\begin{array}{c}>3 \text { Months } \\
\text { (Long-term) } \\
(\mathrm{n}=88)\end{array}$ & $p$-Value \\
\hline 1 & $2.45 \pm 1.63$ & $2.26 \pm 1.59$ & $2.59 \pm 1.67$ & $2.37 \pm 1.61$ & 0.5033 \\
\hline 2 & $1.62 \pm 1.32$ & $1.45 \pm 1.16$ & $1.67 \pm 1.37$ & $1.65 \pm 1.34$ & 0.6725 \\
\hline 3 & $1.91 \pm 1.43$ & $1.95 \pm 1.39$ & $1.92 \pm 1.48$ & $1.88 \pm 1.41$ & 0.9607 \\
\hline 4 & $2.99 \pm 1.60$ & $3.16 \pm 1.64$ & $3.14 \pm 1.63$ & $2.73 \pm 1.53$ & 0.1941 \\
\hline 5 & $2.26 \pm 1.93$ & $2.24 \pm 1.99$ & $2.41 \pm 1.91$ & $2.11 \pm 1.94$ & 0.5929 \\
\hline 6 & $1.67 \pm 1.59$ & $1.76 \pm 1.46$ & $1.72 \pm 1.60$ & $1.57 \pm 1.57$ & 0.7405 \\
\hline 7 & $2.77 \pm 1.59$ & $2.47 \pm 1.57$ & $2.85 \pm 1.63$ & $2.81 \pm 1.56$ & 0.4536 \\
\hline 8 & $2.74 \pm 1.70$ & $2.84 \pm 1.82$ & $2.95 \pm 1.60$ & $2.47 \pm 1.73$ & 0.1727 \\
\hline 9 & $1.32 \pm 1.50$ & $1.24 \pm 1.34$ & $1.44 \pm 1.57$ & $1.22 \pm 1.49$ & 0.5818 \\
\hline 10 & $1.46 \pm 1.43$ & $1.58 \pm 1.27$ & $1.57 \pm 1.56$ & $1.28 \pm 1.34$ & 0.3424 \\
\hline 11 & $0.69 \pm 1.14$ & $0.76 \pm 1.13$ & $0.65 \pm 1.08$ & $0.70 \pm 1.23$ & 0.8605 \\
\hline 12 & $0.82 \pm 1.29$ & $0.97 \pm 1.26$ & $0.92 \pm 1.41$ & $0.63 \pm 1.16$ & 0.2197 \\
\hline 13 & $1.98 \pm 1.73$ & $2.21 \pm 1.66$ & $2.00 \pm 1.75$ & $1.86 \pm 1.74$ & 0.5730 \\
\hline 14 & $1.95 \pm 1.69$ & $1.95 \pm 1.69$ & $1.78 \pm 1.67$ & $2.13 \pm 1.72$ & 0.4022 \\
\hline 15 & $1.77 \pm 1.70$ & $1.95 \pm 1.69$ & $1.62 \pm 1.69$ & $1.86 \pm 1.73$ & 0.5196 \\
\hline 16 & $2.27 \pm 1.52$ & $2.39 \pm 1.60$ & $2.34 \pm 1.51$ & $2.13 \pm 1.50$ & 0.5629 \\
\hline 17 & $2.13 \pm 1.43$ & $2.18 \pm 1.43$ & $2.18 \pm 1.43$ & $2.04 \pm 1.43$ & 0.7606 \\
\hline 18 & $1.93 \pm 1.48$ & $2.00 \pm 1.45$ & $1.97 \pm 1.59$ & $1.84 \pm 1.37$ & 0.8126 \\
\hline 19 & $1.93 \pm 1.45$ & $2.05 \pm 1.45$ & $1.90 \pm 1.53$ & $1.90 \pm 1.38$ & 0.8513 \\
\hline 20 & $1.62 \pm 1.48$ & $1.79 \pm 1.40$ & $1.45 \pm 1.47$ & $1.72 \pm 1.52$ & 0.3504 \\
\hline 21 & $1.46 \pm 1.49$ & $1.71 \pm 1.45$ & $1.37 \pm 1.44$ & $1.46 \pm 1.56$ & 0.4920 \\
\hline 22 & $1.12 \pm 1.40$ & $1.58 \pm 1.41$ & $1.03 \pm 1.37$ & $1.01 \pm 1.41$ & 0.0807 \\
\hline
\end{tabular}

Data are means \pm SD.

Table V. Univariate comparison of patient follow-up regarding preoperative Self-Perception of Nasal Obstruction Score (SPNOS) and mucociliary clearance $(M C C)$.

\begin{tabular}{lcccr}
\hline Measure & Overall & $\begin{array}{c}<1 \text { Month } \\
(\text { Short-term }) \\
(\mathrm{n}=39)\end{array}$ & $\begin{array}{c}1-3 \text { Months } \\
(\text { Medium-term }) \\
(\mathrm{n}=94)\end{array}$ & $\begin{array}{c}>3 \text { Months } \\
(\text { Long-term }) \\
(\mathrm{n}=88)\end{array}$ \\
\hline VAS & & & & \\
Left & $2.60 \pm 1.40$ & $2.71 \pm 1.35$ & $2.59 \pm 1.45$ & $2.55 \pm 1.38$ \\
Right & $2.59 \pm 1.37$ & $2.55 \pm 1.39$ & $2.70 \pm 1.43$ & $2.49 \pm 1.30$ \\
Overall & $2.60 \pm 1.19$ & $2.63 \pm 1.17$ & $2.65 \pm 1.23$ & $2.52 \pm 1.17$ \\
MCC (s) & $1406.64 \pm 516.47$ & $1523.89 \pm 483.51$ & $1343.33 \pm 558.98$ & $1417.46 \pm 482.35$ \\
\hline
\end{tabular}

VAS: Visual Analogue Scale score. Data are means \pm SD.

Regarding compliance after FESS, Lee and colleagues evaluated the ideal frequency for postoperative debridement after FESS and reported that the willingness of patients to comply with postoperative debridement was significantly different based on the frequency of visits for debridement. In their study, patients who were scheduled to have more frequent postoperative debridement reported the greatest disturbances in their academic and socioeconomic activities, as well as more missed/omitted appointments (19). In 2018, Yoo and colleagues evaluated postoperative nasal irrigation practices and their clinical effects on short-term outcomes in patients post-FESS (20). In the study, they found that adherence to irrigation instructions was $82.9 \%$. Factors significantly associated with compliance with irrigation instructions included younger age, prior use of irrigation, revision surgery, and non-native English language speaking (20). Patients were more willing to irrigate with larger volumes if they were younger, had carried out prior irrigation, or had undergone revision surgery (20).

Unlike the studies above, in the current study, we found the critical factor associated with compliance to be age, with older patients being more willing to comply compared 
with younger ones (Table I). In the current study, all patients were well instructed about postoperative care before the surgery. The postoperative follow-up appointments were made after the surgery. However, only $39.8 \%$ of patients were compliant with all regular followup at 3 months after the operation. We identified younger age as the only risk factor for poor compliance with followup throughout the 3 -month period. This finding was different from the previous study conducted by Yoo and colleagues which found that younger patients tend to have higher adherence to sinus irrigation. One possible reason for the discrepancy may be that follow-up appointments require being excused from work and social activities more so than the use of sinus irrigation. On average, younger individuals may participate more frequently in work and social activities. They may be busy and have obligations to job and family. Another reason is the differences in geographic accessibility to medical care between the US (Yoo et al.'s investigated population) and Taiwan (current study). In the US, hospitals are not as near as those in Taiwan for patients to attend for follow-up after FESS. Moreover, hospital density is much higher in Taiwan than that in the USA, meaning patients can visit doctors with less difficulty. The low geographic accessibility may limit older patients in the US in attending follow-up visits to doctors. A third possible driving force for older patients in Taiwan visiting doctors may be that the universal coverage of the health insurance system in Taiwan pays for almost all the fees for everyone to visit doctors, while the financial burden is high for patients visiting doctors in the US.

Limitations of our investigation include the small size of the study population due to the time limit. Except age, no variable was found to be statistically significant out of gender, smoking habit, comorbidity, laterality, Lund-Mackay scoring, atopy, pre-operative SNOT-22, nasal obstruction VAS, and MCC. With more patients enrolled, more factors might be fond that affect the compliance in post-FESS follow-up. Additionally, there may be more factors that should be evaluated, e.g. social economic status, education level and geographic barriers.

\section{Conclusion}

Loss to follow-up is still a common issue in postoperative management of FESS. Regular follow-up after FESS should be as important as the operation itself and might increase the efficacy of the surgical outcomes. Our study identifies older age as being correlated with better compliance in postoperative follow-up specifically in Taiwan. For younger patients, better education and more emphasis on the importance of postoperative follow-up prior to the arrangement of surgery, and paying more attention to their post-operative follow-up are required.

\section{Conflicts of Interest}

All Authors declare that: i) No support, financial or otherwise, has been received from any organization that may have an interest in the submitted work, and ii) there are no other relationships or activities that could appear to have influenced the submitted work.

\section{Authors' Contributions}

Research design: Tsai T and Shih LC; patient and questionnaire summarizing: Shih LC, Hsu CL and Tai CJ; statistical analysis: Lee IT, Ng TY and Wang JY; article writing: Shih LC and Bau DT; reviewing and revising: Tai CJ, Shih LC and Bau DT.

\section{Acknowledgements}

The work was conducted at the Department of Otorhinolaryngology in China Medical University Hospital, and partially supported by the grant from China Medical University Hospital (CMU108-ASIA01) to Dr. Shih and Bau.

\section{References}

1 Slack R and Bates G: Functional endoscopic sinus surgery. Am Fam Physician 58(3): 707-718, 1998. PMID: 9750539.

2 Koskinen A, Salo R, Huhtala H, Myller J, Rautiainen M, Kaariainen J, Penttila M, Renkonen R, Raitiola H, Makela M and Toppila-Salmi S: Factors affecting revision rate of chronic rhinosinusitis. Laryngoscope Investig Otolaryngol 1(4): 96-105, 2016. PMID: 28894807. DOI: 10.1002/lio2.27

3 Khalil HS and Nunez DA: Functional endoscopic sinus surgery for chronic rhinosinusitis. Cochrane Database Syst Rev 19(3): CD004458, 2006. PMID: 16856048. DOI: 10.1002/14651858. CD004458.pub2

4 Eloy P, Andrews P and Poirrier AL: Postoperative care in endoscopic sinus surgery: A critical review. Curr Opin Otolaryngol Head Neck Surg 25(1): 35-42, 2017. PMID: 27846022. DOI: 10.1097/MOO.0000000000000332

5 Jorissen M: Postoperative care following endoscopic sinus surgery. Rhinology 42(30): 114-120, 2004. PMID: 15521662.

6 Rudmik L and Smith TL: Evidence-based practice: postoperative care in endoscopic sinus surgery. Otolaryngol Clin North Am 45(5): 1019-1032, 2012. PMID: 22980682. DOI: 10.1016/j.otc. 2012.06.006

7 Portela RA, Hootnick J and McGinn J: Perioperative care in functional endoscopic sinus surgery: A survey study. Int Forum Allergy Rhinol 2(1): 27-33, 2012. PMID: 22311838. DOI: 10.1002/alr.20098

8 Rudmik L, Soler ZM, Orlandi RR, Stewart MG, Bhattacharyya N, Kennedy DW and Smith TL: Early postoperative care following endoscopic sinus surgery: An evidence-based review with recommendations. Int Forum Allergy Rhinol 1(6): 417-430, 2011. PMID: 22144050. DOI: 10.1002/alr.20072

9 Weber RK and Hosemann W: Comprehensive review on endonasal endoscopic sinus surgery. GMS Curr Top Otorhinolaryngol Head Neck Surg 14: Doc08, 2015. PMID: 26770282. DOI: $10.3205 /$ cto000123

10 Bhattacharyya N: Surgical treatment of chronic recurrent rhinosinusitis: A preliminary report. Laryngoscope 116(10): 
1805-1808, 2006. PMID: 17003725. DOI: $10.1097 / 01 \mathrm{mlg}$. 0000231786.10969.3f

11 Zelle BA, Buttacavoli FA, Shroff JB and Stirton JB: Loss to follow-up in orthopaedic trauma: Who is getting lost to followup? J Orthop Trauma 29(11): 510-515, 2015. PMID: 25866940. DOI: 10.1097/BOT.0000000000000346

12 Hopkins C, Browne JP, Slack R, Lund V and Brown P: The Lund-Mackay staging system for chronic rhinosinusitis: How is it used and what does it predict? Otolaryngol Head Neck Surg 137(4): 555-561, 2007. PMID: 17903570. DOI: 10.1016/ j.otohns.2007.02.004

13 Kennedy JL, Hubbard MA, Huyett P, Patrie JT, Borish L and Payne SC: Sino-nasal outcome test (SNOT-22): A predictor of postsurgical improvement in patients with chronic sinusitis. Ann Allergy Asthma Immunol 111(4): 246-251 e242, 2013. PMID: 24054358. DOI: 10.1016/j.anai.2013.06.033

14 Baroody FM: Mucociliary transport in chronic rhinosinusitis. Clin Allergy Immunol 20: 103-119, 2007. PMID: 17534048.

15 DeConde AS, Mace JC, Bodner T, Hwang PH, Rudmik L, Soler ZM and Smith TL: SNOT-22 quality of life domains differentially predict treatment modality selection in chronic rhinosinusitis. Int Forum Allergy Rhinol 4(12): 972-979, 2014. PMID: 25323055. DOI: 10.1002/alr.21408

16 Juan F, Ayiheng Q, Yuqin F, Hua Z, Jun Y and Bin H: Risk factors of chronic rhinosinusitis after functional endoscopic sinus surgery. Med Sci Monit 23: 1064-1068, 2017. PMID: 28242868. DOI: $10.12659 / \mathrm{msm} .900421$
17 Weber R, Keerl R, Huppmann A, Schick B and Draf W: Effects of postoperative care on wound healing after endonasal paranasal sinus surgery. Laryngorhinootologie 75(4): 208-214, 1996. PMID: 8688126. DOI: $10.1055 / \mathrm{s}-2007-997564$

18 Richtsmeier WJ: Top 10 reasons for endoscopic maxillary sinus surgery failure. Laryngoscope 111(11 Pt 1): 1952-1956, 2001. PMID: 11801976. DOI: 10.1097/00005537-200111000-00015

19 Lee JY and Byun JY: Relationship between the frequency of postoperative debridement and patient discomfort, healing period, surgical outcomes, and compliance after endoscopic sinus surgery. Laryngoscope 118(10): 1868-1872, 2008. PMID: 18641526. DOI: 10.1097/MLG.0b013e31817f93d3

20 Yoo F, Ference EH, Kuan EC, Lee JT, Wang MB and Suh JD: Evaluation of patient nasal saline irrigation practices following endoscopic sinus surgery. Int Forum Allergy Rhinol 8(1): 32-40, 2018. PMID: 29083529. DOI: 10.1002/alr.22034

Received May 28, 2020

Revised June 25, 2020

Accepted June 27, 2020 